\title{
Trial Design of a Prospective Multicenter Diagnostic Accuracy Study of a Point-of-Care Test for the Detection of Taenia solium Taeniosis and Neurocysticercosis in Hospital-Based Settings in Tanzania
}

\author{
Chiara Trevisan ${ }^{1,2, *}++^{(\mathbb{D}}$, Inge Van Damme ${ }^{2,+} \mathbb{D}$, Bernard Ngowi ${ }^{3,4}$, Veronika Schmidt 5,6 (D), Dominik Stelzle $5,7(\mathbb{D}$, \\ Karen Schou Møller ${ }^{8}$, Mwemezi Kabululu ${ }^{9}$ (D), Charles E. Makasi ${ }^{3}$, Pascal Magnussen ${ }^{10}$, Emmanuel Bottieau ${ }^{11}$, \\ Emmanuel Abatih ${ }^{12}$, Maria V. Johansen ${ }^{13}$, Helena Ngowi ${ }^{14}$, Benedict Ndawi ${ }^{15}, \ddagger$, Kabemba E. Mwape ${ }^{16} \mathbb{D}$,

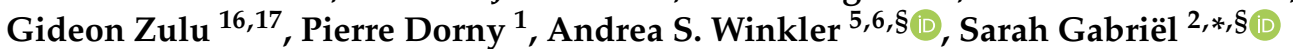 \\ and on behalf of the SOLID Consortium \|
}

check for updates

Citation: Trevisan, C.; Damme, I.V.; Ngowi, B.; Schmidt, V.; Stelzle, D.; Møller, K.S.; Kabululu, M.; Makasi, C.E.; Magnussen, P.; Bottieau, E.; et al. Trial Design of a Prospective Multicenter Diagnostic Accuracy Study of a Point-of-Care Test for the Detection of Taenia solium Taeniosis and Neurocysticercosis in Hospital-Based Settings in Tanzania. Diagnostics 2021, 11, 1528. https:// doi.org/10.3390/diagnostics11091528

Academic Editor: Chao-Min Cheng

Received: 8 July 2021

Accepted: 20 August 2021

Published: 24 August 2021

Publisher's Note: MDPI stays neutral with regard to jurisdictional claims in published maps and institutional affiliations.

Copyright: (c) 2021 by the authors. Licensee MDPI, Basel, Switzerland. This article is an open access article distributed under the terms and conditions of the Creative Commons Attribution (CC BY) license (https:/ / creativecommons.org/licenses/by/ $4.0 /)$.
1 Department of Biomedical Sciences, Institute of Tropical Medicine, 2000 Antwerp, Belgium; pdorny@itg.be 2 Department of Veterinary Public Health and Food Safety, Faculty of Veterinary Medicine, Ghent University, 9820 Merelbeke, Belgium; inge.vandamme@ugent.be

3 National Institute for Medical Research, Muhimbili Medical Research Centre Dar es Salaam, Dar es Salaam 11101, Tanzania; b_ngowi@yahoo.co.uk (B.N.); charlesmakasi2021@gmail.com (C.E.M.)

4 Department of Public Health, University of Dar es Salaam Tanzania, Dar es Salaam 65015, Tanzania

5 Department of Neurology, Center for Global Health, Klinikum rechts der Isar, Technical University of Munich, 81675 Munich, Germany; veronika.schmidt@tum.de (V.S.); dominik.stelzle@tum.de (D.S.); andrea.winkler@tum.de (A.S.W.)

6 Centre for Global Health, Institute of Health and Society, Faculty of Medicine, University of Oslo, 0450 Oslo, Norway

7 Chair of Epidemiology, Department of Sport and Health Sciences, Technical University of Munich, 80809 Munich, Germany

8 Department of Veterinary and Animal Sciences, Faculty of Health and Medical Sciences, University of Copenhagen, 1870 Frederiksberg, Denmark; karenm@sund.ku.dk

9 Tanzania Livestock Research Institute (TALIRI)-Uyole, Mbeya P.O. Box 6191, Tanzania; mwemezie@gmail.com

10 Faculty of Health and Medical Sciences, University of Copenhagen, 2200 Copenhagen, Denmark; pma@sund.ku.dk

11 Department of Clinical Sciences, Institute of Tropical Medicine, 2000 Antwerp, Belgium; EBottieau@itg.be

12 Department of Applied Mathematics, Computer Sciences and Statistics, Faculty of Sciences, Ghent University, 9000 Ghent, Belgium; Emmanuel.Abatih@UGent.be

13 Independent Researcher, 2100 Copenhagen, Denmark; mariavangjohansen@gmail.com

14 Department of Veterinary Medicine and Public Health, College of Veterinary Medicine and Biomedical Sciences, Sokoine University of Agriculture, Morogoro P.O. Box 3021, Tanzania; h_ngowi@yahoo.com

15 Primary Health Care Institute, Iringa 235, Tanzania

16 Department of Clinical Studies, School of Veterinary Medicine, University of Zambia, Lusaka 10101, Zambia; evans.mwape@unza.zm (K.E.M.); gideonzulu@yahoo.com (G.Z.)

17 Eastern Provincial Health Office, Ministry of Health, Lusaka 30205, Zambia

* Correspondence: ctrevisan@itg.be (C.T.); sarah.gabriel@ugent.be (S.G.)

+ Co-first author, these authors contributed equally to this work.

$\ddagger \quad$ Late Dr. Benedict Ndawi passed away on 9 December 2020.

$\S$ Co-last author.

\| SOLID Consortium is listed in the Acknowledgments. 
and a brain computed tomography (CT) scan (stage 2). For groups 2 and 3, a similar design was adopted, but clinical examination and a brain CT scan (stage 2) were only performed in patients who were TS POC test-positive for cysticercosis. Due to the lack of a gold standard, a Bayesian approach was used to determine test accuracy for taeniosis and cysticercosis. For neurocysticercosis, a composite case definition was used as the reference standard. If successful, this study will help the future developments (commercialization and implementation) of the rapid test and improve patient management and disease prevention.

Keywords: Taenia solium; taeniosis; cysticercosis; hospital-based settings; point-of-care test; rapid tests; diagnostics

\section{Introduction}

Taenia solium is a neglected zoonotic parasite contributing to the significant health and economic burden in affected areas of low- and middle-income countries (LMIC) [1].

In Tanzania, several projects on T. solium cysticercosis have been conducted and provided evidence of the parasite presence in several regions of the country [2]. Based on antigen enzyme-linked immunosorbent assay (Ag-ELISA) and antibody (Ab)-ELISA, the prevalence of human cysticercosis (CC) was $16.7 \%$ and $45.3 \%$, respectively, in the Songwe region, southern Tanzania in 2009. In the same area, the prevalence of taeniosis (T) was estimated to be 5.2\% using copro-Ag ELISA [3]. In the Mbulu district, northern Tanzania, a hospital-based brain computed tomography (CT) scan study indicated that almost $20 \%$ of people with epilepsy suffered from neurocysticercosis (NCC) [4,5].

At the district hospital level, the diagnosis of tapeworms can be performed using microscopy, but has a low sensitivity and fails to differentiate Taenia spp. Other methods, such as the copro-antigen ELISA, the recombinant antigen-based enzyme-linked immunoelectrotransfer blot (EITB), and polymerase chain reaction (PCR) techniques are available but also have limitations in their performance, and are used only in research settings as laboratories at the district hospital level in Tanzania lack equipment, adequately trained experts, and infrastructure [6]. Moreover, differential diagnosis and the stigma associated with epilepsy further contribute to the challenges that district hospitals face [7].

To standardize the diagnosis of NCC, Del Brutto et al. [8] formulated a set of criteria based on neuroimaging, assisted by serological, clinical, and epidemiological results. Neuroimaging is possible using CT and/or magnetic resonance imaging (MRI). For serology, the lentil lectin-bound glycoproteins-enzyme-linked immunoelectrotransfer blot (LLGPEITB) is the test of choice [8,9]. In resource-poor areas, this is problematic as access to neuroimaging is very limited, trained neurologists are scarce, and the LLGP-EITB, though considered as having high specificity and sensitivity [10], is expensive and in a format not applicable/available in most local laboratories. Therefore, disease diagnosis in resourcepoor areas outside a research setting is hampered by the lack of good tools and skilled personnel, as well as the high costs.

To limit these challenges, a user-friendly, rapid, cheap T. solium point-of-care test (TS POC) was developed by the Centre for Disease Control and Prevention, Atlanta, USA, in collaboration with the Klinikum rechts der Isar, Technical University of Munich, Germany.

The main objective of the SOLID project ("Evaluation of an antibody-detecting pointof-care test for the diagnosis of Taenia solium taeniosis and neurocysticercosis in communities and primary care settings of highly endemic, resource-poor areas in Tanzania and Zambia, including the training of-and technology transfer to the Regional Reference Laboratory and health centers") was to evaluate the sensitivity/specificity and ease of use of the TS POC test for the detection of T. solium T and NCC in resource-poor, highly endemic areas in sub-Saharan Africa. The study in Zambia was conducted in a community-based setting and its design was published elsewhere [11]. This paper focuses on the design of the diagnostic accuracy evaluation of the TS POC test at the district hospital level in Tanzania. 


\section{Materials and Methods}

\subsection{TS POC Test}

A detailed description of the TS POC test is presented in [11]. To summarize, the TS POC test is an antibody-detecting prototype developed by a team of researchers from the USA and Germany. The TS POC test consists of a double-strip cassette that holds one strip for the detection of antibodies against the adult stage (using the recombinant $\mathrm{T}$ antigen rES33), and one to detect antibodies against the larval stage of the parasite (using the recombinant CC antigen $\mathrm{rT24H}$ ) [12,13]. Each strip has one test line, one control line, and a separate port for the sample application, enabling the simultaneous detection of $\mathrm{T}$ and $\mathrm{CC}$ antibodies.

For the TS POC test evaluation in Tanzania, 3000 tests were produced at CDC Atlanta in collaboration with Arista Biologicals Inc, Allentown, PA, USA.

Details on how the TS POC test was performed and read can be found in [14]. In essence, two times 20 micro-liters of blood are collected from a fingertip using a micropipette and placed on the sample well for T and CC, respectively. Immediately after, the chase buffer is applied, and as soon as the flow commences the timer is set for $20 \mathrm{~min}$ (Figure 1). The TS POC test is read by two independent readers (two-factorial design), and in the case of a disagreement, a third reader intervenes. A result card is used to report the results which are subsequently entered in electronic case report forms (eCRFs). In the case of an invalid result (e.g., no flow, or control line missing), the test is repeated once.

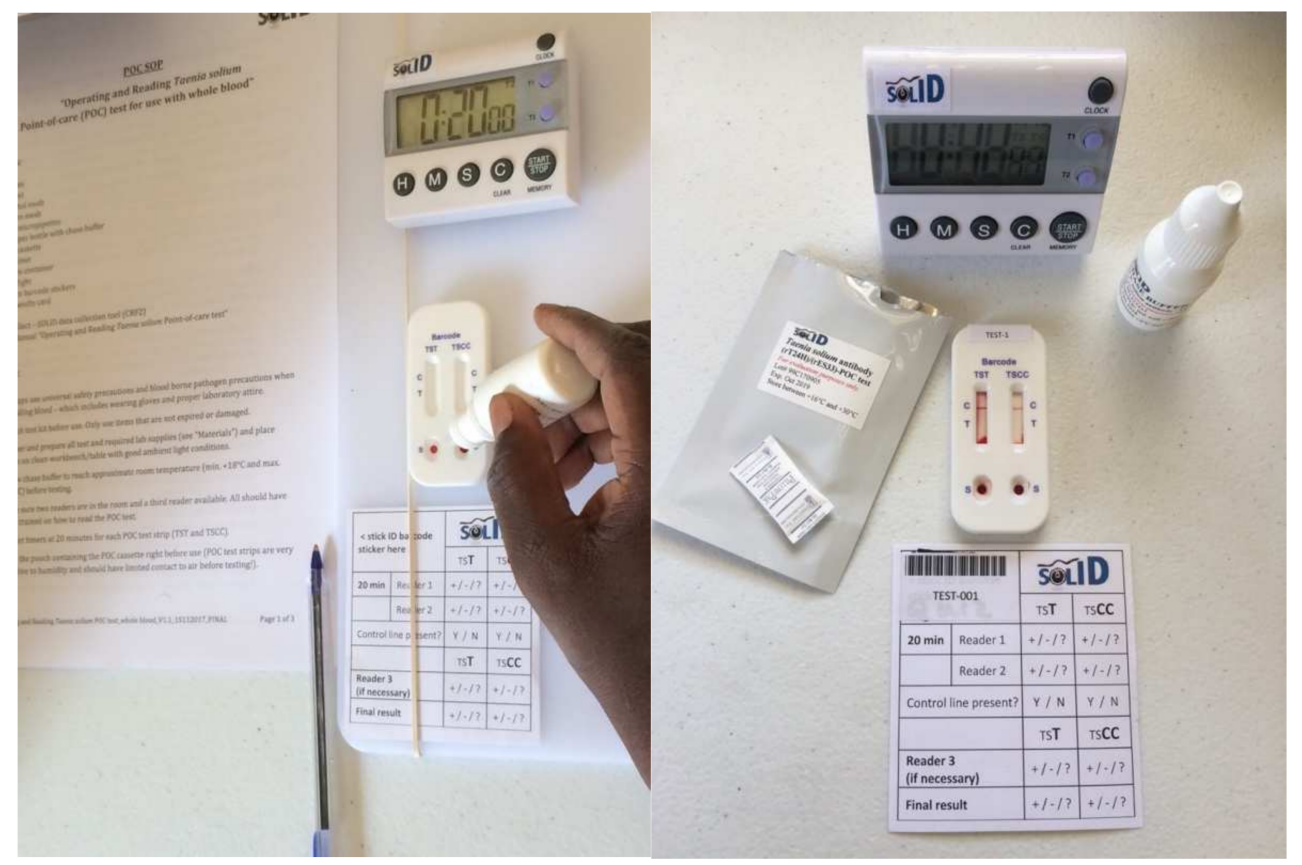

Figure 1. Photos showing the TS POC test, chase buffer, timer set on $20 \mathrm{~min}$, and TS POC result card.

\subsection{Study Design, Recruitment, and Eligibility}

To evaluate the diagnostic performance of the TS POC test for the detection of T, CC, and NCC, a prospective, two-stage, multicenter study was conducted. The TS POC test was evaluated at the stage where it may be implemented in practice, being patients attending district hospitals in T. solium-endemic areas. Since the accuracy of the test was likely to vary across subgroups of patients [15], the study was performed on three groups of patients, namely patients with specific neurological signs and symptoms (epilepsy and/or severe progressive chronic headache) (group 1); patients with complaints compatible with intestinal worm infections (group 2); patients with other symptom(s) (group 3). To get a representative sample for the studied populations, subjects for groups 1 and 2 were consecutively recruited, while for group 3, only every tenth patient was included if eligibility criteria were met. Recruitment took place between December 2017 and February 2020. 
Patients of group 1 were recruited from the mental health ward or the outpatient department while patients belonging to groups 2 and 3 were recruited from the outpatient department only. To be included, patients had to be ten years old or above and willing and able to participate in all aspects of the study (including providing blood and stool samples, undergoing a clinical examination, and a brain CT scan, if necessary), willing and able to provide written informed consent (assent for minors with consent from a parent or a legally authorized representative), living in the study area for the past three months and planning to stay in the same area throughout the study period. Pregnant women and patients suffering from severe health conditions needing in-patient care were excluded from the study.

To be eligible for inclusion in group 1, patients needed to present to the mental health ward or outpatient department with on-going symptoms of severe progressive chronic headache impeding their daily activities without fever and signs of cerebral infection or other obvious causes; and/or they should have a history of one or more epileptic seizures without obvious causes. Participants meeting any of the following criteria were not enrolled in the study: less than ten years of age; acute febrile illness; fever and signs and symptoms for middle ear infection; signs and symptoms of meningitis; neck muscle pain and stiffness; a history of stroke with neurological focal deficit; a history of psychiatric problems.

To be eligible for inclusion in group 2, patients had to present at the outpatient department with intestinal complaints suggestive of an intestinal worm infection (diarrhea and/or abdominal pain/discomfort for more than two weeks and/or a recent history of worm expulsion). For group 3, eligible patients with any other symptom(s) not immediately linked with intestinal worm infections or symptoms described for group 1 were also recruited from the outpatient department.

\subsection{Study Objectives and Outcomes}

The primary objective of the study was to assess the sensitivity and specificity of the TS POC test for the diagnosis of each of the disease presentations (T, CC, and NCC). Due to the lack of a gold standard, three different reference tests were used for the detection of $\mathrm{T}$ and CC, respectively (Table 1). The diagnostic accuracy of the TS POC test for the detection of $\mathrm{T}$ was determined against two stool-based reference methods (copro antigen ELISA and copro PCR) and a serological test (immunoblot using the recombinant protein rES33). For CC, the reference methods were the EITB-LLGP, rT24H immunoblot, and the B158/B60 monoclonal antibody-based serum antigen ELISA. For NCC, a composite case definition was used as the gold standard based on Del Brutto's criteria [8]. An overview of the different reference tests included is given in Table 1 , and a description of the tests is presented in Section 2.6.

Table 1. Reference tests used to assess the sensitivity and specificity of the TS POC test for the detection of taeniosis, cysticercosis, and neurocysticercosis.

\begin{tabular}{cccc}
\hline Disease Presentation & TS POC Test Strip & Purpose & Reference Standard \\
\hline Taeniosis & TS POC T & $\begin{array}{c}\text { Diagnosis/treatment } \\
\text { Monitoring/surveillance } \\
\text { Epidemiological research }\end{array}$ & $\begin{array}{c}\text { copro antigen ELISA (stool ag) } \\
\text { copro PCR (stool DNA) } \\
\text { rES33 immunoblot (serum ab) }\end{array}$ \\
Cysticercosis & TS POC CC & $\begin{array}{c}\text { Monitoring/surveillance } \\
\text { Epidemiological research }\end{array}$ & $\begin{array}{c}\text { EITB-LLGP (serum ab) } \\
\text { rT24H immunoblot (serum ab) } \\
\text { serum antigen ELISA (serum ag) }\end{array}$ \\
\hline Neurocysticercosis & TS POC CC & $\begin{array}{c}\text { Diagnosis/treatment and } \\
\text { management }\end{array}$ & $\begin{array}{c}\text { Composite case definition } \\
\text { according to Del Brutto et al.'s } \\
\text { criteria [8] }\end{array}$ \\
\hline
\end{tabular}

TS POC T: Taenia solium point-of-care test taeniosis test strip; ELISA: enzyme-linked immunosorbent assay; PCR: Polymerase Chain Reaction; TS POC CC: Taenia solium point-of-care test cysticercosis strip; EITB: enzyme-linked immunoelectrotransfer blot; LLGP: lentil lectin-bound glycoproteins. 


\subsection{Study Area}

The study was conducted in three rural district hospitals in the Songwe and Mbeya regions, Tanzania. District hospitals were included based on the following eligibility criteria: (i) presence of a mental health ward; (ii) proximity to an area where T and NCC were prevalent; (iii) closeness to the Mbeya Radiology Centre equipped with a CT scanner. The three district hospitals in Mbozi (Vwawa), in rural Mbeya (Ifisi), and in Rungwe (Tukuyu) were included (Figure 2).

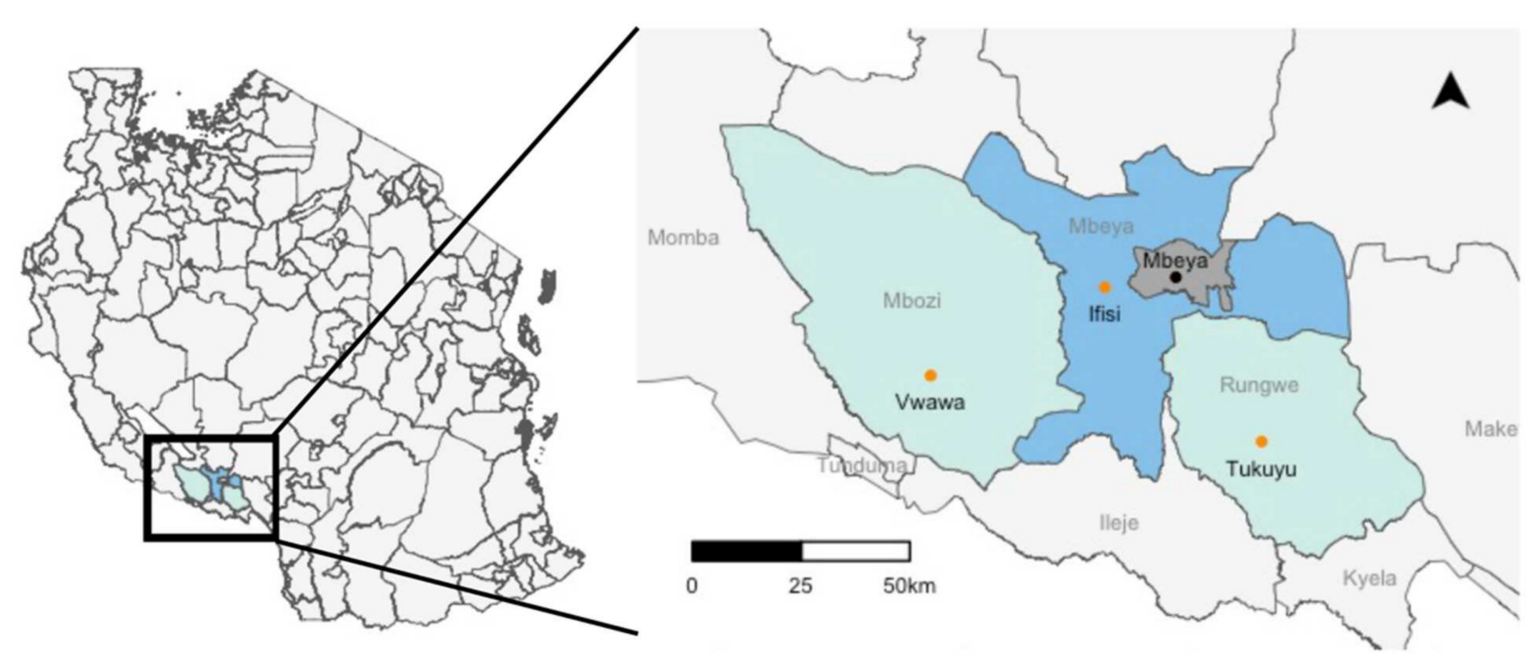

Figure 2. Map of Tanzania showing the study area and a close-up of the district hospitals (orange dots) in Mbozi (Vwawa), rural Mbeya (Ifisi), and Rungwe (Tukuyu), and the CT scan facility in Mbeya (black dot) in the Mbeya and Songwe regions in Tanzania.

\subsection{Study Workflow}

After enrollment, patients were subjected to the TS POC test, and based on its results, a subset of participants was subjected to several reference tests, clinical examination, and CT scanning of the brain. Per hospital, approximately one-third of the required sample size was enrolled (see Section 2.11). The study design flow for group 1 is visualized in Figure 3, while Figure 4 shows the study design flow for groups 2 and 3.

\subsection{Reference Sample Collection, Processing, and Analysis}

Sample collection, processing, shipment, and analysis were the same as for the SOLID project community-based study in Zambia [11]. To summarize, $3 \mathrm{~mL}$ of venous blood was collected, and the serum was stored at $-20^{\circ} \mathrm{C}$ until reference testing using the LLGP-EITB, recombinant immunoblots (rES33 and rT24H), and Ag ELISA. Stool samples were stored at room temperature in 10\% formalin for copro-Ag-ELISA, and 70\% ethanol for copro PCR.

The samples were shipped and analyzed in Belgium. Most analyses were performed at the Institute of Tropical Medicine in Antwerp, Belgium, with a 20 percent subset of samples analyzed at Ghent University, Belgium, for quality control. Only the serum samples for the Ag ELISA were fully analyzed at Ghent University, with a 20 percent subset of samples analyzed at the Institute of Tropical Medicine in Antwerp. 


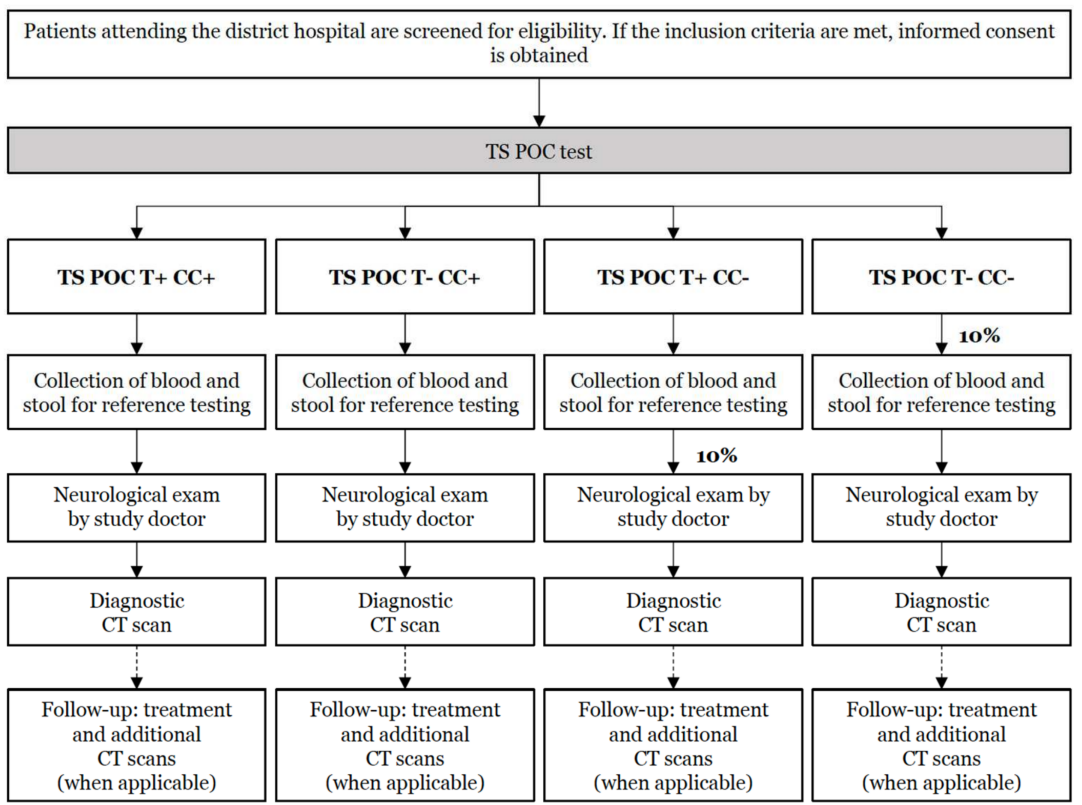

Figure 3. Flow of the diagnostic accuracy study in Tanzania for patients attending the mental health ward or outpatient department of district hospitals with on-going symptoms of severe progressive chronic headache impeding their daily activities without fever and signs of cerebral infection or other obvious causes; and/or a history of one or more epileptic seizures without obvious causes (group 1). In this two-stage design, all patients were tested using the TS POC test (=index test; stage 1), after which all positive, and a subset of negative, patients were selected for different reference tests, clinical examination, and a brain CT scan (stage 2).

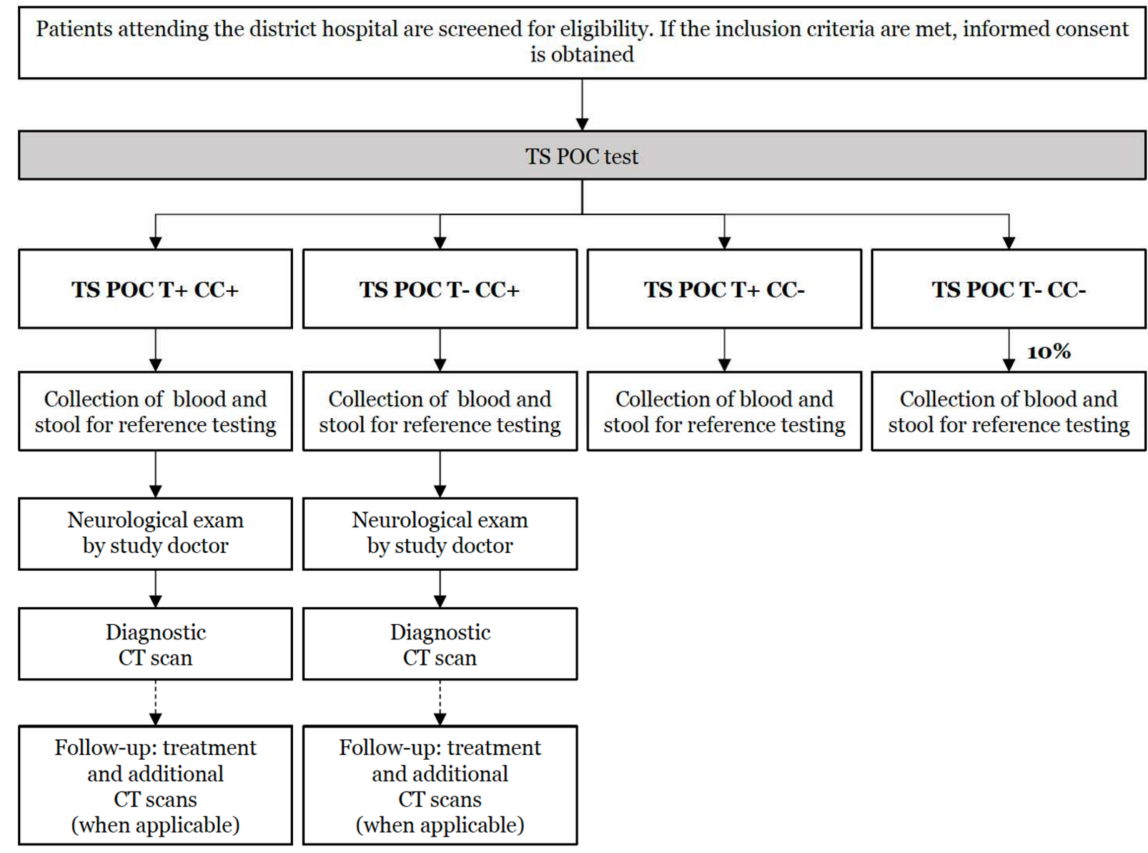

Figure 4. Flow of the diagnostic accuracy study in Tanzania for patients attending the outpatient department of district hospitals with symptoms of intestinal worm infections (group 2) and other symptoms (group 3) that were not specific neurological signs and symptoms. In this two-stage design, all patients were tested using the TS POC test (=index test; stage 1), after which all positive, and a subset of negative, patients were selected for different reference tests. Clinical examination and a brain CT scan (stage 2) were only performed on patients positive for CC on the TS POC test. 
In short, the LLGP-EITB was performed as described previously $[10,16]$ with a few modifications. The LLGP-EITB strip was considered positive if at least one out of seven bands were visible. For the recombinant strips, rES33 and rT24H lines for T and CC were combined in one strip. All strips were provided by CDC. The B158/B60 serum antigen ELISA was used as previously described [17]. For the Copro-Ag-ELISA, the procedure was performed as reported previously by [18] and modified by [19]. DNA extraction of stool samples $\left(200 \mu \mathrm{L}\right.$ in $70 \%$ ethanol) was carried out using the QIAmp ${ }^{\circledR}$ fast DNA stool mini kit (Qiagen, Hilden, Germany) according to the manufacturer's instructions. DNA was stored at $-20^{\circ} \mathrm{C}$ until use. The copro PCR was performed according to [20] with some modifications. A sample was only considered positive if T. solium DNA was detected in the sample.

\subsection{Clinical Follow-Up, CT Scans, and Patient Management}

A procedure as described for the community-based study in Zambia was followed [11]. For group 1, all patients were seen by a doctor (Figure 2) who performed a neurological examination, while for groups 2 and 3 (Figure 3) only study participants TS POC CCpositive were seen by a study doctor. Appropriate eCRFs were used to record patients' personal and clinical data according to principles of good clinical practices. After the neurological examination, including past and current medical history, a cerebral CT scan with and without contrast was taken. Contrast was used only if there were no clinical contraindications. If two or more months between the initial TS POC test result and the CT scan elapsed, a new TS POC test was performed using only the strip for CC.

The CT scan results were read independently by the senior project neuroradiologist and an NCC expert. For each CT scan, the number, location, and stage of the lesion were recorded. In the case of a discrepancy, a third independent reviewer adjudicated the CT diagnosis. The final NCC diagnosis was determined according to the 2017 criteria of [8]. These were based on clinical symptoms, neuroimaging, the detection of antibodies and antigens in serum, and epidemiological considerations corresponding to absolute, major, and minor criteria and resulting in the definite and probable diagnosis of NCC. An absolute neuroimaging criterion for NCC was at least one cystic lesion showing the scolex. Major neuroimaging criteria were cystic lesions without scolex, single or multiple rings, or nodular enhancing lesions, multilobulated cystic lesions in the subarachnoid space, and parenchymal brain calcifications. Hydrocephalus or abnormal enhancement of basal leptomeninges were categorized as minor neuroimaging criteria. Four different stages of lesions were defined: vesicular, colloidal vesicular, granular nodular, and nodular calcified. The first three were considered as active NCC lesions and the last was defined as an inactive NCC lesion [8].

\subsection{Patient Treatment and Follow-Up}

Treatment with niclosamide (single oral dose, $2 \mathrm{~g}$ ) or praziquantel $(10 \mathrm{mg} / \mathrm{kg})$ (depending on the availability of the drugs) was offered to patients who tested positive for $\mathrm{T}$ using the TS POC T or any of the reference tests. Relatives of these patients were also informed and invited for a diagnostic test, and in the case of positivity, treatment was offered.

Patients who underwent a CT scan were informed about the results during a follow-up appointment, and management procedures as described for the community-based study in Zambia were followed [11]. In short, if indicated, anthelmintic treatment was offered according to national guidelines and Winkler et al. If indicated, anthelmintic treatment with albendazole ( $15 \mathrm{mg} / \mathrm{kg} / \mathrm{d}$ ) was offered for 10 days, accompanied with corticosteroid treatment (dexamethasone $12 \mathrm{mg}$ to $20 \mathrm{mg} / \mathrm{d}$ ), starting one day before and ending three days after anthelmintic treatment with half the dosage. Dexamethasone dosage depended on the location and number of cysticerci [21]. Symptomatic NCC cases with active lesions were treated with anthelminthic medication and steroids for two weeks according to the patient's response. Treatment was tailored to each individual patient. At set time points (after six 
weeks and again after six months), a follow-up clinical examination was performed. During these time points, follow-up CT scans were offered to monitor treatment success.

\subsection{Training}

Training and capacity building were cornerstones of the project. Extensive multiple full-day training sessions were organized at the district hospitals to ensure the collection of high-quality data and correct patient management and follow-up. Three-day training sessions were arranged where everyone involved in the project was trained. Sessions on parasite biology, diagnosis, treatment, prevention, and control of the parasite in endemic areas were organized for all project staff members.

Tailored training was organized for clinicians responsible for recruiting patients, nurses responsible for obtaining informed consent and TS POC testing, and laboratory technicians responsible for blood and stool sample collection and processing. Medical doctors responsible for clinical examinations, including the neurological examination and later patient follow-ups and treatment were trained by the neurology team, contributing to capacity building in the study area. Assessments and follow-up refresher training were planned to assure the correct following of study procedures and good clinical (laboratory) practices and sample collection. Monitoring visits to assess study conduct and compliance on good storage of TS POC tests, samples, and other study material were conducted twice a month. If deviations from standard operating procedures were encountered, corrective measures were initiated.

\subsection{Data Management}

To ensure the collection of high-quality data, all people involved in patient recruitment and performing the POC test were trained and followed up regularly by project members. Non-confidential data were collected electronically on tablets using EpiCollect5 (@) 2021 Centre for Genomic Pathogen Surveillance, v4.2.0, https: / / five.epicollect.net, accessed on 8 July 2021). Specific CRFs were created, and real-time data validation rules were included whenever possible. More details can be found in [11].

Laboratory technicians performing the reference tests were blinded to the TS POC results. Reading of the CT scans was carried out by two independent readers, both blinded to the TS POC and reference tests results. Discrepancies between two readers were identified and solved by a third reader, who was also blinded to the TS POC results.

\subsection{Sample Size and Statistical Analysis}

The sample size for group 1 was calculated to obtain the desired half-width of $5 \%$ around the $95 \%$ confidence interval for sensitivity and specificity [22]. Assuming a sensitivity of $93 \%$, a specificity of $99 \%$ [11], and a prevalence of NCC of $20 \%, 500$ participants were required. To account for losses, $20 \%$ were added to this number. The final sample size for group 1 was set at 600 .

Since the prevalence of $\mathrm{T}$ in the study population was unknown, the sample size for groups 2 and 3 was calculated for different plausible values, ranging between $3 \%$ and $10 \%$. Assuming a sensitivity of $82 \%$, a specificity of $81 \%$, and the desired precision of $10 \%$, 1890 participants were required. To account for $5 \%$ contingencies, the final sample size was rounded to 2000 patients.

The sensitivity and specificity of the TS POC test for the detection of T, CC, and NCC were determined relative to the reference tests as shown in Table 1, for each of the three study groups. Weighting was used to correct for the study design (a subset of T-CC that is selected for sampling/clinical examination). The positive and negative predictive values were also determined.

For $\mathrm{T}$ and $\mathrm{CC}$, the diagnostic sensitivity and specificity of the TS POC test were estimated using a Bayesian approach as described previously [23]. For the evaluation of the TS POC test performance for NCC, sensitivity and specificity were computed by 
cross-tabulating POC results with those of the final NCC diagnosis. More details can be found in [11].

\section{Discussion}

This trial was designed to provide a rigorous evaluation of TS POC test accuracy for the detection of T, CC, and NCC. The ambitious four-year project was the result of longstanding North-South collaborations and aimed at including 2600 patients of three district hospitals in Tanzania. If the test is validated with success, it may be proposed for commercialization and implementation at district hospitals and community health centers in endemic areas.

As several sources of bias can arise in diagnostic accuracy studies [24], several measures were taken to assure the internal validity of our study. As unblinded studies may have a higher sensitivity and overall accuracy of results when the person interpreting the reference standard is aware of the index test result [25], diagnostic review bias in the present study was avoided by blinding all laboratory technicians to the TS POC test result. Moreover, as the interpretation of several reference test results for $\mathrm{T}$ and $\mathrm{CC}$ have inherent test integrity (such as ELISA results, which are read by a spectrophotometer), diagnostic review bias seemed very unlikely for the evaluation of T and CC. On the other hand, clinical examination, and interpretation of CT scans, which were both used for the composite case definition for NCC, are more subjective. Therefore, a structured questionnaire was used to record the results of the neurological examinations to reduce subjectivity in the examinations as much as possible. Moreover, all CT scans were read in detail by two independent readers, and in the case of a disagreement, additionally by a third independent reader, who were all blinded to the TS POC result.

Stool and serum samples for the reference tests were requested immediately after performing the TS POC test to reduce loss-to-follow-up and to avoid disease progression bias. Although we aimed to perform the CT scans with minimal delay, the CT scans could not always be performed within a reasonable time frame after the index test due to several logistical reasons (such as an inoperative CT scanner and travel planning of the neurology team to be present when CT scans were taken). Since delayed verification may result in biased accuracy estimates [25], an additional TS POC test was performed on patients for whom more than two months had passed between the initial TS POC test and CT scanning. These results were used to assess the extent of disease progression bias.

As disease severity and participant demographics can influence test accuracy, the TS POC test evaluation in the SOLID project included different groups of patients attending the district hospitals for which the test may be used in practice. Moreover, to increase generalizability, three different district hospitals were chosen in the design. To obtain a representative sample of the different studied populations, patients attending the outpatient department (and the mental health ward for patients with specific neurological signs and symptoms) were recruited consecutively for groups 1 and 2, and selecting every tenth patient in group 3 . Although several efforts were made to increase the external validity of the study, results may not be generalizable to patients attending local healthcare facilities or other more specialized hospitals, where differences in disease spectrum or other underlying (demographic) factors might be present.

If successful, the tool will be the first POC test contributing to NCC diagnosis which could be made available at the district hospital level. Combined with increased knowledge, training, and capacity building, this project aided staff at the district hospital level to identify, correctly and early, suspected NCC cases. Furthermore, thanks to the training, staff will be able to make the connections between NCC on the one side and epileptic seizures/epilepsy, severe progressive chronic headache, and other neurological signs/symptoms on the other side. Finally, we do hope that patients will be referred to neuroimaging facilities or more specialized hospitals when necessary. This will lead to improved patient management practices, contributing to a positive effect on the patients' health outcome, and raising awareness of T. solium-associated neurological diseases, e.g., 
epileptic seizures/epilepsy, which bear an enormous burden of the disease, especially in LMIC [26]. NCC is one of the main causes of epileptic seizures/epilepsy in T. soliumendemic areas [27] and it is a curable disease. A POC test clearly has the potential to contribute to changing the narrative around epilepsy being a chronic disease with often high mortality in LMIC [26] to a curable disease in T. solium-endemic areas. Additionally, a correct diagnosis and knowledge of NCC will potentially stimulate the people's perception of epilepsy as being a biological disease (e.g., NCC as its cause) rather than a consequence of witchcraft, which is a common belief in the study area [28]. Creating awareness and educating professionals in disease recognition will further lead to an increased number of detected cases. If the TS POC test proves to have acceptable sensitivity and specificity to detect NCC cases, further studies are required to evaluate the TS POC test's ability to guide clinical decision-making [29].

The TS POC test will be the first portable, easy-to-apply diagnostic test for $\mathrm{T}$ available at the district hospital level. This will lead to the early detection and treatment of tapeworm carriers. The latter is crucial to reduce/stop disease transmission as tapeworm carriers are responsible for environmental contamination with infective eggs. These carriers represent a risk not only for themselves but also for their close contacts. Early detection and treatment will ensure a rapid halt to this environmental contamination, reducing the risk of new NCC cases and the infection of pigs, and thus, of perpetuating the life cycle. The training will guide the staff in the treatment and follow-up of tapeworm carriers at the household and community level.

Adequate monitoring and surveillance systems, which are necessary to support the sustainable control of T. solium, are currently not implemented in sub-Saharan Africa, largely due to the lack of well-performing tests that are deployable in low-resource settings. Therefore, as well as its diagnostic use, the TS POC may also be used for monitoring and surveillance purposes, as it is affordable, relatively rapid, equipment-free, and userfriendly [14]. For this aim, the TS POC test was also validated for use at the community level in Zambia, which mainly included asymptomatic people [11].

In Tanzania, in contrast to Zambia, the study was conducted primarily by trained hospital staff, and recruitment and testing occurred daily until the target sample size was obtained. To assure the smooth conduct of the study and the correct following of study standard operating procedures, the intensive training of staff involved in the study was essential. In the SOLID project, new digital tools for data collection with barcoding systems were introduced, which might have posed a challenge to members of staff more used to traditional data recording methods. However, the digitalization of data collection was chosen for the SOLID project to be able to monitor study progress by assessing the number of entries uploaded, to assist staff when issues might appear during regular data checks, cleaning, and validation sessions performed by project members, on top of advancing technological knowhow in resource-limited areas. To assure high-quality data collection, correct patient follow-up, and adequate sample collection, processing and storage, the district hospital staff was monitored by project members' fortnightly visits.

\section{Conclusions}

In conclusion, if the test is successfully validated, the new, rapid, cheap TS POC test might be produced and used in various settings of T. solium-endemic areas and assist in diagnosing, monitoring and surveillance, and the epidemiological research of $T$. solium taeniosis/NCC. This will be important for triaging patients for treatment, especially those who suffer from epileptic seizures/epilepsy. In this context, it is important to mention that the development of the WHO NCC management guidelines is in its final stages. Their implementation will rely on validated diagnostic tools, such as the TS POC test. Hence, our study is timely and, depending on the outcome, will contribute to the successful implementation of the WHO NCC management guidelines. Moreover, the intensive training and follow-up visits contributed to capacity building at the district hospital level and increased awareness about the disease and potential management, treatment, and 
follow-up of affected patients. In turn, this has potential benefits for close contacts, families, and surrounding communities.

Author Contributions: Conceptualization, S.G., P.D., V.S., A.S.W., B.N. (Bernard Ngowi), K.E.M., P.M., H.N., G.Z., E.B., E.A. and M.V.J.; Project coordinator and study coordinator, P.D.; Initiated the study, S.G.; Funding acquisition, S.G., P.D., A.S.W., H.N., V.S., P.M., M.V.J. and K.E.M.; Methodology, S.G., C.T., I.V.D., K.E.M., V.S., P.M., G.Z., C.E.M., D.S., E.B., E.A., M.V.J., A.S.W. and P.D.; The protocols for recruitment, POC testing, management/processing of samples, lab analyses, and clinical examinations were developed in close collaboration between NIMR (B.N. (Bernard Ngowi), B.N. (Benedict Ndawi)), UNZA (K.E.M., G.Z.), ITM (C.T., P.D., E.B.), TUM (V.S., D.S., A.S.W.), and UGent (S.G., I.V.D.); The data management team was composed of project members of UGent (I.V.D., E.A.); Before the start of the study, training of study staff was conducted by senior project members with long standing experience in African field settings, (A.S.W., P.M., G.Z., K.E.M., E.B., S.G., C.T., M.V.J., V.S., B.N. (Bernard Ngowi), B.N. (Benedict Ndawi), K.S.M. and K.E.M.); Treatment and follow-up protocols of patients with NCC were developed jointly by TUM (D.S., A.S.W.), NIMR (B.N. (Bernard Ngowi), UNZA (G.Z.), UCPH (P.M.), and ITM (E.B.); Managed the production, technical and logistical transfer of the TS POC test and the immunoblot reference tests to the study partners, V.S.; Project administration, S.G. and C.T.; Supervision: S.G., C.T., B.N. (Benedict Ndawi), B.N. (Bernard Ngowi), A.S.W., P.D. and P.M. Visualization, C.T. and I.V.D.; Writing-original draft preparation, C.T., I.V.D. and S.G.; Writing-review and editing, I.V.D., C.T., B.N. (Bernard Ngowi), V.S., D.S., K.S.M., M.K., C.E.M., P.M., E.B., E.A., M.V.J., H.N., B.N. (Benedict Ndawi), K.E.M., G.Z., P.D., A.S.W. and S.G. All authors have read and agreed to the published version of the manuscript.

Funding: This research was funded by the European and Developing Countries Clinical Trials Partnership (EDCTP) (grant number DRIA2014-308) and the German Federal Ministry of Education and Research (BMBF) (grant number: 01KA1617) within the research grant "Evaluation of an antibody detecting point-of-care test for the diagnosis of Taenia solium taeniosis, and (neuro)cysticercosis in communities and primary care settings of highly endemic, resource-poor areas in Tanzania and Zambia, including training of-and technology transfer to the Regional Reference Laboratory and health centers (SOLID)".

Institutional Review Board Statement: The study was conducted according to the guidelines of the Declaration of Helsinki, and was granted ethical clearance for the Tanzanian part of the study by the National Ethics Health Research Committee (NatREC) of Tanzania (NIMR/HQ/R.8a/Vol.IX/2597), the Institute of Tropical Medicine (IRB/AB/ac/112 Ref 1177/17) through the ethics committee of the University of Antwerp (EC UZA 17/31/352), and the Technical University of Munich through their Ethics Committee at the Klinikum rechts der Isar, Munich (299/18S). Authorization to conduct research was also granted by the National Health Research Authority at the Ministry of Health in Zambia.

Informed Consent Statement: Informed consent was obtained from all subjects involved in the study.

Data Availability Statement: No new data were created or analyzed in this manuscript. Data sharing is not applicable to this article.

Acknowledgments: John Noh, Holly Chastain, and Sukwan Handali from the Division of Parasitic Diseases and Malaria, Center for Global Health, Centers for Disease Control and Prevention (CDC), Atlanta, GA, USA, are acknowledged for helping with the compilation of the TS POC kits, development of the test manual, and handling of the logistics at the study sites. We acknowledge all members of the SOLID consortium who are not included in the author list of this paper: Ana Lucia Fajardo Castaneda, Anke Van Hul, Charlotte Rüther, Chishala Chabala, Chishimba Mubanga, Dries Reynders, Famke Jansen, Isaac K. Phiri, Maxwell Masuku, Richard Mambo, Rune Stensvold, and Sandra Vangeenberghe. We thank all the staff for their hard work and the participants for their willingness to participate. We also wish to acknowledge members of the SOLID External Advisory Board for their advice. Finally, all members of the SOLID consortium dedicate this work to the memory of our late colleague and friend, Benedict Ndawi.

Conflicts of Interest: V.S. was involved in the development of the TS POC test. The Technical University of Munich owns the intellectual property of the TS POC test. The Institute of Tropical Medicine (Nationalestraat 155, B-2000 Antwerp, Belgium) is the sponsor of the study. The funders 
had no role in the design of the study; in the collection, analyses, or interpretation of data; in the writing of the manuscript, or in the decision to publish the results.

\section{References}

1. Trevisan, C.; Devleesschauwer, B.; Schmidt, V.; Winkler, A.S.; Harrison, W.; Johansen, M.V. The societal cost of Taenia solium cysticercosis in Tanzania. Acta Trop. 2017, 165, 141-154. [CrossRef] [PubMed]

2. Ngowi, H.A.; Winkler, A.S.; Braae, U.C.; Mdegela, R.H.; Mkupasi, E.M.; Kabululu, M.L.; Lekule, F.P.; Johansen, M.V. Taenia solium taeniosis and cysticercosis literature in Tanzania provides research evidence justification for control: A systematic scoping review. PLoS ONE 2019, 14, e0217420. [CrossRef] [PubMed]

3. Mwanjali, G.; Kihamia, C.; Kakoko, D.V.; Lekule, F.; Ngowi, H.; Johansen, M.V.; Thamsborg, S.M.; Willingham, A.L. Prevalence and Risk Factors Associated with Human Taenia Solium Infections in Mbozi District, Mbeya Region, Tanzania. PLoS Negl. Trop. Dis. 2013, 7, e2102. [CrossRef] [PubMed]

4. Blocher, J.; Schmutzhard, E.; Wilkins, P.P.; Gupton, P.N.; Schaffert, M.; Auer, H.; Gotwald, T.; Matuja, W.; Winkler, A.S. A crosssectional study of people with epilepsy and Neurocysticercosis in Tanzania: Clinical characteristics and diagnostic approaches. PLoS Negl. Trop. Dis. 2011, 5, e1185. [CrossRef] [PubMed]

5. Winkler, A.S.; Blocher, J.; Auer, H.; Gotwald, T.; Matuja, W.; Schmutzhard, E. Epilepsy and neurocysticercosis in rural TanzaniaAn imaging study. Epilepsia 2009, 50, 987-993. [CrossRef]

6. Mwape, K.E.; Gabriël, S. The Parasitological, Immunological, and Molecular Diagnosis of Human Taeniasis with Special Emphasis on Taenia solium Taeniasis. Curr. Trop. Med. Rep. 2014, 1, 173-180. [CrossRef]

7. Winkler, A.S.; Willingham, A.L.; Sikasunge, C.S.; Schmutzhard, E. Epilepsy and neurocysticercosis in sub-Saharan Africa. Wien. Klin. Wochenschr. 2009, 121, 3-12. [CrossRef] [PubMed]

8. Del Brutto, O.H.; Nash, T.E.; White, A.C.; Rajshekhar, V.; Wilkins, P.P.; Singh, G.; Vasquez, C.M.; Salgado, P.; Gilman, R.H.; Garcia H.H. Revised diagnostic criteria for neurocysticercosis. J. Neurol. Sci. 2017, 372, 202-210. [CrossRef]

9. Deckers, N.; Dorny, P. Immunodiagnosis of Taenia solium taeniosis/cysticercosis. Trends Parasitol. 2010, 26, 137-144. [CrossRef] [PubMed]

10. Tsang, V.C.; Brand, J.A.; Boyer, A.E. An enzyme-linked immunoelectrotransfer blot assay and glycoprotein antigens for diagnosing human cysticercosis (Taenia solium). J. Infect. Dis. 1989, 159, 50-59. [CrossRef]

11. Van Damme, I.; Trevisan, C.; Mwape, K.E.; Schmidt, V.; Magnussen, P.; Zulu, G.; Mubanga, C.; Stelzle, D.; Bottieau, E.; Abatih, E.; et al. Trial Design for a Diagnostic Accuracy Study of a Point-of-Care Test for the Detection of Taenia solium Taeniosis and (Neuro)Cysticercosis in Community Settings of Highly Endemic, Resource-Poor Areas in Zambia: Challenges and Rationale. Diagnostics 2021, 11, 1138. [CrossRef] [PubMed]

12. Levine, M.Z.; Calderón, J.C.; Wilkins, P.P.; Lane, W.S.; Asara, J.M.; Hancock, K.; Gonzalez, A.E.; Garcia, H.H.; Gilman, R.H.; Tsang, V.C. Characterization, cloning, and expression of two diagnostic antigens for Taenia solium tapeworm infection. J. Parasitol. 2004, 90, 631-638. [CrossRef]

13. Hancock, K.; Pattabhi, S.; Whitfield, F.W.; Yushak, M.L.; Lane, W.S.; Garcia, H.H.; Gonzalez, A.E.; Gilman, R.H.; Tsang, V.C. Characterization and cloning of T24, a Taenia solium antigen diagnostic for cysticercosis. Mol. Biochem. Parasitol. 2006, 147, 109-117. [CrossRef] [PubMed]

14. Mubanga, C.; Mwape, K.E.; Phiri, I.K.; Trevisan, C.; Kabululu, M.; Zulu, G.; Van Damme, I.; Schmidt, V.; Dorny, P.; Gabriël, S. Operational characteristics of an antibody detecting point of care test for Taenia solium infections in a community and hospital setting. BMC Infect. Dis. 2021, 21, 607. [CrossRef] [PubMed]

15. Rutjes, A.W.; Reitsma, J.B.; Vandenbroucke, J.P.; Glas, A.S.; Bossuyt, P.M. Case-control and two-gate designs in diagnostic accuracy studies. Clin. Chem. 2005, 51, 1335-1341. [CrossRef]

16. Noh, J.; Rodriguez, S.; Lee, Y.M.; Handali, S.; Gonzalez, A.E.; Gilman, R.H.; Tsang, V.C.; Garcia, H.H.; Wilkins, P.P. Recombinant protein- and synthetic peptide-based immunoblot test for diagnosis of neurocysticercosis. J. Clin. Microbiol. 2014, 52, 1429-1434. [CrossRef]

17. Dorny, P.; Phiri, I.K.; Vercruysse, J.; Gabriel, S.; Willingham, A.L.; Brandt, J.; Victor, B.; Speybroeck, N.; Berkvens, D. A Bayesian approach for estimating values for prevalence and diagnostic test characteristics of porcine cysticercosis. Int. J. Parasitol. 2004, 34, 569-576. [CrossRef]

18. Allan, J.C.; Avila, G.; Garcia Noval, J.; Flisser, A.; Craig, P.S. Immunodiagnosis of taeniasis by coproantigen detection. Parasitology 1990, 101 Pt 3, 473-477. [CrossRef]

19. Mwape, K.E.; Phiri, I.K.; Praet, N.; Muma, J.B.; Zulu, G.; van den Bossche, P.; de Deken, R.; Speybroeck, N.; Dorny, P.; Gabriël, S. Taenia solium infections in a rural area of Eastern Zambia-A community based study. PLoS Negl. Trop. Dis. 2012, 6, e1594. [CrossRef]

20. Yamasaki, H.; Allan, J.C.; Sato, M.O.; Nakao, M.; Sako, Y.; Nakaya, K.; Qiu, D.; Mamuti, W.; Craig, P.S.; Ito, A. DNA differential diagnosis of taeniasis and cysticercosis by multiplex PCR. J. Clin. Microbiol. 2004, 42, 548-553. [CrossRef]

21. Winkler, A.S. Neurocysticercosis in sub-Saharan Africa: A review of prevalence, clinical characteristics, diagnosis, and management. Pathog. Glob. Health 2012, 106, 261-274. [CrossRef]

22. Banoo, S.; Bell, D.; Bossuyt, P.; Herring, A.; Mabey, D.; Poole, F.; Smith, P.G.; Sriram, N.; Wongsrichanalai, C.; Linke, R.; et al. Evaluation of diagnostic tests for infectious diseases: General principles. Nat. Rev. Microbiol. 2008, 6, S16-S28. [CrossRef] 
23. Berkvens, D.; Speybroeck, N.; Praet, N.; Adel, A.; Lesaffre, E. Estimating disease prevalence in a Bayesian framework using probabilistic constraints. Epidemiology 2006, 17, 145-153. [CrossRef] [PubMed]

24. Whiting, P.F.; Rutjes, A.W.; Westwood, M.E.; Mallett, S.; Deeks, J.J.; Reitsma, J.B.; Leeflang, M.M.; Sterne, J.A.; Bossuyt, P.M. QUADAS-2: A revised tool for the quality assessment of diagnostic accuracy studies. Ann. Intern. Med. 2011, 155, 529-536. [CrossRef]

25. Whiting, P.F.; Rutjes, A.W.; Westwood, M.E.; Mallett, S. A systematic review classifies sources of bias and variation in diagnostic test accuracy studies. J. Clin. Epidemiol. 2013, 66, 1093-1104. [CrossRef] [PubMed]

26. Knauss, S.; Stelzle, D.; Emmrich, J.V.; Korsnes, M.S.; Sejvar, J.J.; Winkler, A.S. An emphasis on neurology in low and middle-income countries. Lancet. Neurol. 2019, 18, 1078-1079. [CrossRef]

27. Carabin, H.; Ndimubanzi, P.C.; Budke, C.M.; Nguyen, H.; Qian, Y.; Cowan, L.D.; Stoner, J.A.; Rainwater, E.; Dickey, M. Clinical manifestations associated with neurocysticercosis: A systematic review. PLoS Negl. Trop. Dis. 2011, 5, e1152. [CrossRef] [PubMed]

28. Winkler, A.S.; Mayer, M.; Schnaitmann, S.; Ombay, M.; Mathias, B.; Schmutzhard, E.; Jilek-Aall, L. Belief systems of epilepsy and attitudes toward people living with epilepsy in a rural community of northern Tanzania. Epilepsy Behav. 2010, 19, 596-601. [CrossRef] [PubMed]

29. Rodger, M.; Ramsay, T.; Fergusson, D. Diagnostic randomized controlled trials: The final frontier. Trials 2012, 13, 137. [CrossRef] 